\title{
Neuere Ergebnisse zur zeitlichen Einstufung der spätglazialen Moränenstände und zum Verlauf der postglazialen Waldgrenze im nordöstlichen Pelvoux (französische Nordalpen)
}

\author{
1. Die geomorphologischen Grundzüge \\ des Untersuchungsgebietes unter besonderer \\ Berücksichtigung der spätglazialen \\ Vergletscherungsphasen
}

Das Untersuchungsgebiet umfaßt im wesentlichen nordwestliche Randbereiche des Pelvoux-Massivs östlich der bekannten Wintersportstation Les Deux-Alpes. Hier wurden in einem ehemaligen natürlichen See, dem $L a c$ du Plan, 1982 mehrere fossile Hölzer geborgen. Aus glazialmorphologischen Gründen wurde noch das von cou. TEAUX pollenanalytisch untersuchte Tal der Pisse de la Muzelle', das wenig südlich von Les Deux-Alpes liegt, mit in die Betrachtungen einbezogen.

Der die fossilen Hölzer bergende Lac du Plan liegt in knapp $2300 \mathrm{~m}$ rund $3 \mathrm{~km}$ östlich von Les Deux-Alpes in Gneisen, an die sich ein Streifen dunkler, liasischer Kalkschiefer aus dem Domérien-Toarcien im Osten anschließt. Seine Entstehung verdankt der See dem glazialen Schurf im Kristallin, in dem er zur Gänze angelegt ist. Der obere Einzugsbereich des Ruisseau du Grand Plan ist heute nicht mehr vergletschert. Theoretisch müßten die hochgelegenen Gratflanken jedoch noch im 19. Jahrhundert von Gletschern eingenommen worden sein. Möglicherweise sind die verwaschenen Moränen nordöstlich der Tete Moute dem Stand von 1850 zuzuordnen. In der Combe du Thuit, $400 \mathrm{~m}$ westlich des Lac du Plan, ist ein markantes dreigegliedertes spätglaziales Moränensystem entwickelt. Es setzt in $2240 \mathrm{~m}$ an und bricht in $2010 \mathrm{~m}$ ab. Der 2:1-Methode wie der Lichtenecker-Visser-Methode gemäß hat sich dafür eine stadiale Schneegrenze von 2240 bis $2250 \mathrm{~m}$ und damit ein am ehesten daunzeitliches Alter ergeben. Nach den bemerkenswerten pollenanalytischen Ergebnissen von cOUTEAUX (1983a) im Pisse de la Muzelle-Tal müßte diese daunzeitliche Moräne älter als das Bölling, ja sogar älter als zumindestens die auslaufende Älteste Dryas sein. Denn cou. TEAUX (1982a, S. 37) fand in der Tourbière de la Muzelle, in $2140 \mathrm{~m}$ Höhe, in maximal $7 \mathrm{~m}$ mächtigen Ton-, Gyttjaund Torfablagerungen eine komplette Abfolge ältestdryaszeitlicher bis subatlantischer Pollensequenzen. Dieses Ergebnis beweist, dass die Höhenbereiche, in dem sich das dreigestaffelte Moränensystem der Combe de Thuit befindet, seit der auslaufenden Ältesten Dryas nicht mehr vergletschert gewesen sein können und die hier beobachteten Daunmoränen demnach älter als das Bölling sein müssen. $\mathrm{Zu}$ ähnlichen Ergebnissen bezüglich des Daunalters kam BURGA (1981, S. 149) im Bereich des Albulapasses.

Die rezente Schneegrenze bezogen auf 1978 im Pisse de la Muzelle-Tal liegt nach der 2:1-Methode bei $2715 \mathrm{~m}$ und nach der Höfer-Methode bei $2830 \mathrm{~m}$. Die 1850erSchneegrenze erreichte hier nach der 2:1-Methode $2585 \mathrm{~m}$ (= Depression von $130 \mathrm{~m}$ ), nach der Höfer-Methode $2721 \mathrm{~m}$ (= Depression von $109 \mathrm{~m}$ ) und nach der Lichtenecker-Visser-Methode $2565 \mathrm{~m}$ (= Depression von $150 \mathrm{~m}$ ) auf die nach der 2:1-Methode berechnete rezente Schneegrenze von 1978. Der sich daraus ergebende durchschnittliche Depressionswert für den 1850er Stand entspricht in etwa den Werten, die auch für die benachbarte Belledonne errechnet worden sind (HANNSS, 1982, Tabelle 15).

Geht man von den von MAISCH (1987, S. 69; 1982, S. 101) mitgeteilten Depressionswerten für die spätglazialen Gletscherstände aus, die größenordnungsmäßig auch für die französischen Alpen gelten dürften (HANNSS, 1982, S. $131 ; 1980$, S. 190), so würden sich im Pisse de la Muzelle Tal für das Egesen $(=-215 \mathrm{~m})$ eine theoretische stadiale Schneegrenze von $2355 \mathrm{~m}$, für das Daun $(=-310 \mathrm{~m})$ von $2260 \mathrm{~m}$, für das Gschnitz $(=-670 \mathrm{~m})$ von $1940 \mathrm{~m}$ und für den Steinach-Stand $(=-800 \mathrm{~m})$ von 1770 m ergeben. Das würde bedeuten, dass das Gschnitz- und Steinach-Stadium auf alle Fälle älter als das Bölling bzw. mindestens die auslaufende Jüngere Dryas sind, da laut den pollenanalytischen Ergebnissen von COUTEAUX (1982a, S. 37) seit der (oberen?) Jüngeren Dryas im Pisse de la Muzelle-Tal zumindestens alle Areale unterhalb von 2140 m, der Höhe, auf der sich die Tourbière de la Muzelle entwickelt hat, eisfrei geblieben sein müssen.

J. Biju-Duval, Direction Départementale de l'Agriculture, 42. rue Marcelin Berthelot, F-38040 Grenoble

Ch. Hannss, Geographisches Institut, Universität Tübingen, Hölderlinstr. 12, D-7400 Tübingen

W. Schoch, Labor für quartäre Hölzer, Tobelhof $13, \mathrm{CH}-8134$ Adliswil

S. Wegmüller, Botanisches Institut der Universität Bern, Altenbergrain 21, $\mathrm{CH}-3013$ Bern 

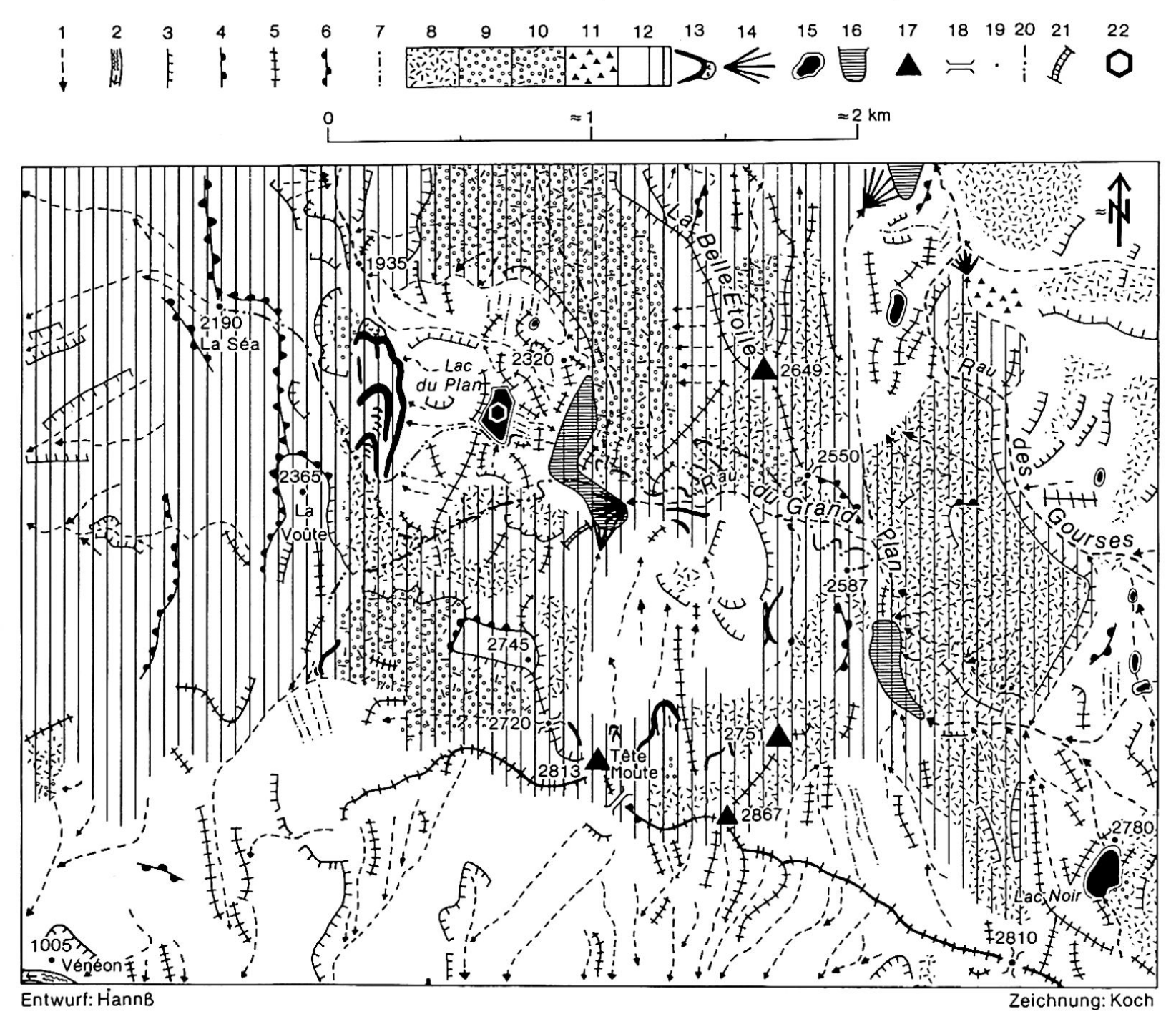

Abb. 1: Die geomorphologischen Grundzüge im Bereich des Lac du Plan (nach Geländebeobachtungen und Luftbildinterpretation der Aufnahmen F 813003363 214, 215 und 216; HANNSS)

\author{
1 = Fluviatil entstandene Tiefenlinien \\ 2 = Flußlauf \\ $3=$ Kante \\ $4=$ Abgerundete Kante \\ $5=$ Grat, Rippe \\ $6=$ Rücken \\ $7=$ Kluftlinie \\ 8 = Sturzhalden, unbewachsener Schutt \\ $9=$ Begrünte Halde \\ $10=$ Absterbende Halde, teilweise begrünt \\ $11=$ Felssturzmaterial \\ $12=$ Gneise (weiß) bzw. mesozoische Sedimentgesteine, \\ vor allem liasische Schiefer und Kalke \\ (= Senkrechtschraffur)
}

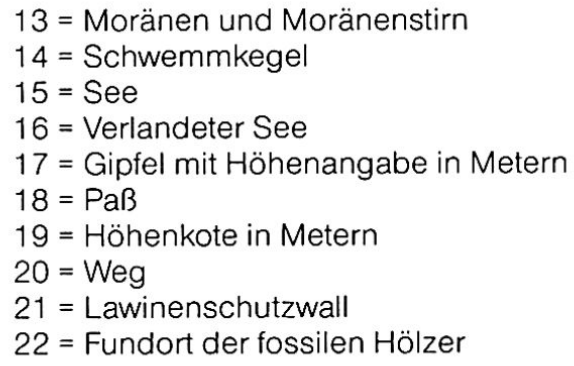

$14=$ Schwemmkegel

$16=$ Verlandeter See

$17=$ Gipfel mit Höhenangabe in Metern

$18=\mathrm{Pa} B$

$20=$ Weg

22 = Fundort der fossilen Hölzer

* Für die Mitarbeit an den Reinzeichnungen der drei Abbildungen sei herzlich Herrn G. KOCH vom Geographischen Institut der Universität Tübingen gedankt. 
Aber auch der Daunstand müßte auf Grund der allgemeinen Reliefverhältnisse und der von COUTEAUx hier erarbeiteten Pollendiagramme noch in die Zeit vor das Bölling zu stellen sein, da die theoretische Daunschneegrenze aufder Grundlage der von MAISCH 1987 publizierten Depressionswerte mit $310 \mathrm{~m}$ unter dem 1850erHochstand nur $120 \mathrm{~m}$ über der Tourbière de la Muzellegelegen haben dürfte. Wenn der daunzeitliche Gletschervorstoß erst in der Älteren Dryas stattgefunden hätte, wie das PATZELT und BORTENSCHLAGER annehmen (1978, S. 186), dann wäre mit ziemlicher Sicherheit die Tourbière de la Muzelle noch einmal vom Eis überfahren und wären die bis in die Älteste Dryas zurückreichenden Moorablagerungen wieder ausgeräumt worden. Dies ist aber nicht der Fall.

Nur der Egesen-Stand sollte auch nach den im oberen Pisse de la Muzelle-Tal angetroffenen Verhältnissen jünger als das spätglaziale Bölling-Alleröd-Interstadial sein. Denn ein Gletscher mit der Schneegrenzdepression von 215 m hätte nach der 2:1-Methode im Ruisseau de Chapeau Roux-Ast theoretisch nur bis in rund $2150 \mathrm{~m}$ herab gereicht und damit die spätglazialen Ablagerungen der Tourbière de la Muzelle unberührt gelassen. Ein postallerödzeitliches Alter der Egesen-Moränen postuliert für die Schweizer Alpen überdies BURGA (1981, S. 149).

Das bisher Gesagte gilt weitgehend auch, wenn man die spätglazialen stadialen Schneegrenzen für das Tal der Pisse de la Muzelle nicht nach den von MAISCH 1987 und 1982 errechneten Depressionswerten bestimmt, sondern die in der nahegelegenen Belledonne von HANNSS (1982, S. 131) gewonnenen absoluten Schneegrenzhöhen der vier spätglazialen Hauptstände Steinach $=\mathrm{Sp}-\mathrm{A}=$ $2010 \mathrm{~m}$, Gschnitz $=$ Sp-B $=2120 \mathrm{~m}$, Daun $=\mathrm{Sp}-\mathrm{C}=$ $2330 \mathrm{~m}$ und Egesen $=\mathrm{Sp}-\mathrm{D}=2240 \mathrm{~m}$ verwendet.

Die zeitliche Einstufung der wichtigsten spätglazialen Gletschervorstöße auf der Basis der von COUTEAUX im Pisse de la Muzelle erzielten pollenanalytischen Ergebnisse ist eine Bestätigung der in den letzten Jahren von der Zürcher Geomorphologenschule erarbeiteten Spätglazialchronologie (MAISCH, 1987, S. 65-71) und widerspricht der von PATZELT (PATZELT, BORTENSCHLAGER, 1978, S. 195) geäußerten Ansicht, daß der Daunstand nicht bereits in die Älteste, sondern erst in die Mittlere Dryas gehöre. Diese Ansicht wurde auch auf die französischen Alpen übertragen (DE BEAULIEU, JORDA, 1977, S. 13; HANNSS, 1980, S. 190). Sie wird heute hier jedoch nicht mehr vertreten (BOREL et al., 1984, S. 45, 50, 51; JORDA, 1987, S. 66).

Wenn aber nun aller Wahrscheinlichkeit nach nicht nur der Steinach-, Gschnitz- und Clavadelvorstoß, sondern auch der des Daun bereits vor dem Bölling-Interstadial stattgefunden hat, ist es auf Grund der pollenanalytisch abgesicherten starken Abkühlung während der Jüngsten Dryas sehr unwahrscheinlich, daß die EgesenMoränen nicht schon während dieses jüngsten spätglazialen Stadials, sondern erst im Präboreal abgelagert worden sein sollen, so wie das DE BEAULIEU, JORDA (1977, S. 13) und BOREL et al. (1984, S. 50) vorgeschlagen haben. Dem widerspricht überdies die ausgeprägte und plötzliche Kli- maverbesserung seit dem beginnenden Präboreal, wenn man dafür die in den Westalpen erarbeiteten Ergebnisse als Argumentationsgrundlage heranzieht (BURGA, 1987, S. 76; WEGMULLER, 1977, S. 163).

\section{Die heutigen Vegetationsverhältnisse}

Nach TONNEL, OZENDA (1964, Karte nach S. 24) ist das Waldkleid im Raum von Les Deux-Alpes durch folgende Stufen von unten nach oben gekennzeichnet: Auf den Schatthängen des Vénéon- und RomancheTales nördlich und südlich von Les Deux-Alpes greifen Fichtenbestände bis rund $1700 \mathrm{~m}$ nach oben, die noch der montanen Stufe zugeordnet werden. Darüber folgen Pinus uncinata- Formationen. Der Vegetationskarte von TONNEL, OZENDA gemäß reichen sie westlich und östlich von Les Deux-Alpes bis in die Höhen von 2300 m. Darüber erstreckt sich die alpine Stufe.

Nach der 1983 in allen Einzelheiten von SCHWARZ aufgezeigten, auf der Frosttrocknistheorie beruhenden Methode, wurde von ihm freundlicherweise die heutige potentielle obere Baumgrenze im Bereich von Les Deux-Alpes errechnet. Als klimatische Referenzstation diente ihm das in $1470 \mathrm{~m}$ Höhe nördlich des Lac du Chambon gelegene Besse ${ }^{2}$. Es ergab sich ein Wert von $2200 \mathrm{~m}$, und zwar sowohl unter Verwendung des interdiurnen Veränderlichkeitswertes von 1 wie von 2.

Die reale heutige Wald- und Baumgrenze liegt jedoch um Les Deux-Alpes fast durchweg unter $2000 \mathrm{~m}$. Nördlich des Lac du Plan, im oberen Einzugsbereich des Ruisseau de la Pisse, reicht die Waldgrenze stellenweise sogar bis $1700 \mathrm{~m}$ nach oben. Die Talhänge westlich und östlich von Les Deux-Alpes sind heute nahezu waldfrei. Einzelne Gehölze, die bis $2170 \mathrm{~m}$ hinaufreichen, zeigen jedoch an, daß die natürliche Waldgrenze hier anthropogen stellenweise um mindestens 500 m erniedrigt worden sein muß. Auf große Diskrepanzen zwischen der rezenten Waldund Baumgrenze hat COUTEAUX (1983c, S. 72) im Vallon de Lavey hingewiesen, einem westlichen Nebenast des Vénéon-Tales, rund $10 \mathrm{~km}$ SSWlich des Lac du Plan. Die reale Waldgrenze liegt ihm zu Folge heute hier bei nur $1580 \mathrm{~m}$. Juniperus communis kommt im Vallon de Lavey vereinzelt dagegen noch bis $1830 \mathrm{~m}$, Picea abies (L.) bis $1880 \mathrm{~m}$, Populus tremula L. und Sorbus aucuparia L. bis $2000 \mathrm{~m}$, Betula pendula Roth bis $2040 \mathrm{~m}$ und Pinus cembra $L$ sogar noch in $2150 \mathrm{~m}$ vor. Es ist interessant, daß die Geländebefunde im Bereich von Les Deux-Alpes und im Vallon de Laveyziemlich genau mit der von SCHWARZ theoretisch berechneten heutigen realen Baumgrenze von $2200 \mathrm{~m}$ übereinstimmen.

\section{Die fossilen Hölzer und Torfe des Lac du Plan}

Daß zwischen 4800 B. P. und 3920 B. P., also im Jüngeren Atlantikum und älteren Subboreal, Baum-, wenn nicht sogar Waldwuchs im Bereich des nordwestlichen Pelvoux-Massivs bis in mindestens $2300 \mathrm{~m}$ Höhe möglich 


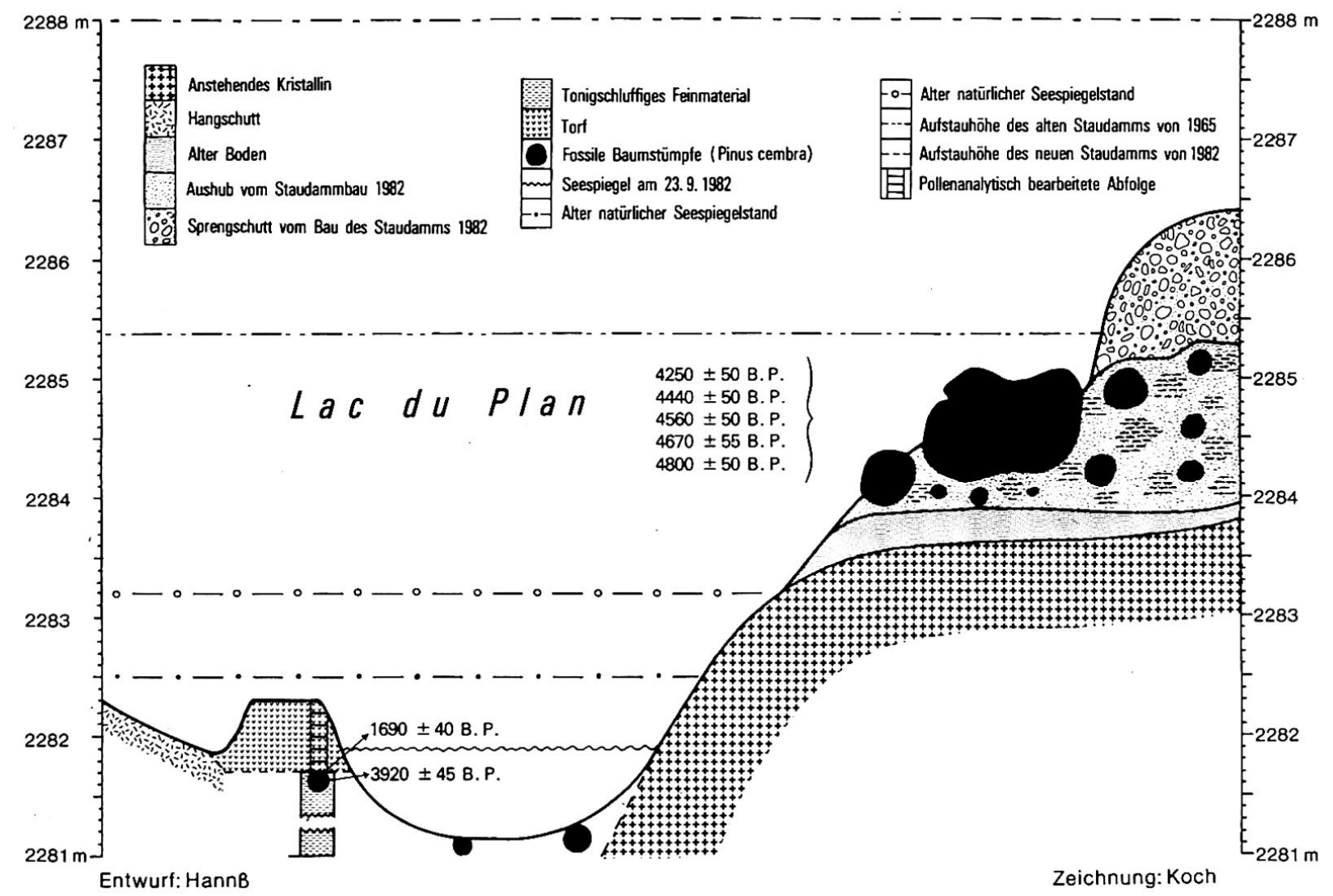

Abb. 2: Die fossilen Arvenhölzer im Lac du Plan (nach Geländeaufnahmen; HANNSS und BIJU-DUVAL)

war, zeigen die fossilen Arvenhölzer (Pinus cembra) bzw. die Makroreste von Alnus spec. und Salix spec., die 1982 im Lac du Plan geborgen worden sind.

Nach COUTEAUX (1984, S. 58, 1983c, S. 74, 76) wurden zudem im bereits erwähnten Vallon de Lavey in dem hier in $2050 \mathrm{~m}$ Höhe und damit $470 \mathrm{~m}$ über der heutigen realen Waldgrenze gelegenen Moor La Muande 8 Stücke von $P i$ nus silvestris, 1 Stück eines Pinus spec. sowie Rindenstücke von Betula gefunden. Nach dem gleichen Autor (1984, S. 58) sind diese Hölzer zwischen $5150 \pm 200$ B.P. und $2570 \pm 170$ B. P. alt. Es wurde laut COUTEAUX (1983c, S. 74) zudem im gleichen Moor ein 2570 B. P. altes Pinus un-
cinata-Fragment und der Rest einer 2320 B. P. alten Pinus cembra geborgen. Letztere würden also bereits aus dem frühen Älteren Subatlantikum stammen. Dem gleichen Autor gemäß (COUTEAUX, 1983c, S. 74, 76) wurden im Vallon de Lavey in $2120 \mathrm{~m}$ fossile Reste von Alnus viridis und Pinus cembra sowie in $1900 \mathrm{~m}$ und $1830 \mathrm{~m}$ Höhe solche von Pinus cembra und Pinus uncinata beobachtet. Die in $1830 \mathrm{~m}$ Höhe geborgenen fossilen Holzfragmente stammen aus dem Mittelalter. Nach COUTEAUX (1983c, S. $78,85)$ ist im Vallon de Lavey in jüngerer Zeit jedoch nicht die Wald-, sondern nur die Baumgrenze anthropogen stark erniedrigt worden, und zwar insbesondere im 14. und 19. Jahrhundert, als der Bevölkerungsdruck hier

Tabelle 1: ${ }^{14} \mathrm{C}$-Daten der fossilen Arven (Pinus cembra), und der Torfe in Jahren B. P. aus dem Lac du Plan (2285 m) ${ }^{3}$

\begin{tabular}{|llll|}
\hline Alter & $\begin{array}{l}\text { Probenummer } \\
\text { der }{ }^{14} \text { C-Labors }\end{array}$ & $\begin{array}{l}\text { Eigene } \\
\text { Probenummer }\end{array}$ & $\begin{array}{l}\text { Datiertes } \\
\text { Material }\end{array}$ \\
\hline $1690+40$ & HD 9968-9963 & 1717 & Torf \\
$3920 \pm 45$ & HD 9967-9962 & 1804 & Pinus cembra \\
$4250 \pm 50$ & HD 9019-9440 & 1836 & Pinus cembra \\
$4440 \pm 50$ & HD 9020-9441 & 1847 & Pinus cembra \\
$4560 \pm 50$ & HD 8094-8206 & 1846 & Pinus cembra \\
$4670 \pm 55$ & HD 9018-9426 & 1835 & Pinus cembra \\
$4800 \pm 50$ & HD 8358-8912 & 1848 & Pinus cembra \\
\hline
\end{tabular}


am stärksten war und durch die Köhler viele Solitärbäume oberhalb der Waldgrenze geschlagen worden sind.

Die fossilen Arven im Lac du Plan wurden 1982 geborgen, als der alte Stausee aus dem Jahr 1965 entleert worden ist. Die Torfe auf der Ostseite des Lac du Plan zeigen an, daß er postglazial zeitenweise sogar noch unter den niedrigsten, auf Abb. 2 eingetragenen natürlichen Seespiegelstand abgesunken sein muß. Die pollenanalytische Untersuchung des kurzen Profilabschnittes von nur $57 \mathrm{~cm}$ Gesamtmächtigkeit umfaßt 6 Torfproben. Es handelt sich ausschließlich um Cyperaceentorf. Die Aufbereitung der Proben erfolgte am Systematisch-Geobotanischen Institut der Universität Bern nach der üblichen Methode $(\mathrm{HCl}, \mathrm{KOH}, \mathrm{HF}, \mathrm{HCl}$, Acetolyse, $\mathrm{KOH}$, Glycerin). Hier standen zur Analyse auch die Vergleichssammlung rezenter Pollen und die zahlreichen Pollenphotos zur Verfügung.

Die Bezugssumme zur Berechnung der prozentualen Anteile der einzelnen Taxa umfaßt die Summe der ausgezählten Baum- und Nichtbaumpollen ohne die Cyperaceen. Die Sporen wurden ebenfalls nicht in die Bezugssumme einbezogen.

Zur Darstellung der Kurven des Diagramms wurde ein einheitlicher Maßstab, für die Cyperaceen hingegen ein logarithmischer Maßstab verwendet. Werte unter 0,5\% wurden durch kleine Kreisflächen dargestellt.

Die verhältnismäßig hohen Nichtbaumpollen-Anteile (35-69\%) des Profils legen den Schluß auf eine Waldgrenzsituation der Mulde zur Zeit der Torfablagerung nahe. Die geringen Anteile von Abies (Weißtanne) von nur $1-13 \%$, die aus dem Fernflug stammen dürften, weisen rückläufige Tendenz auf. Picea (Fichte) hält sich ziemlich konstant, wenn auch mit gedrückten Werten (10\%). Pinus (Kiefer) dürfte im jüngeren Zeitabschnitt eine leichte Ausbreitung erfahren haben. Juniperus (Wacholder) fügt sich gut ins Bild der Waldgrenzsituation ein. Alnus viridis (Grünerle) ist mit 3,5-8\% verhältnismäßig gut vertreten, geht dann aber im Zuge der Kiefernausbreitung zurück. In der Nähe der Mulde dürften am Ende der Torfablagerung Kiefernbestände mit etwas Fichten, in feuchten schattigen Runsen Grünerlengebüsche verbreitet gewesen sein.

Oberhalb von $27 \mathrm{~cm}$ Profiltiefe steigen die Cerealia-Werte (Fernflug) bis zu $4 \%$ an. Ferner stellen sich auch Spuren von Zeigern der Weiderodung (Artemisia, Chenopodiaceae, Plantago lanceolata) aus tieferen Lagen (?) ein. Bemerkenswert ist zudem das Einsetzen von Juglans (Nußbaum) in $17 \mathrm{~cm}$ Tiefe. In allen untersuchten Proben fanden sich außerdem Pollen der Ericaceen (VacciniumTyp), die als Anzeichen einer Verstrauchung und auch der Versauerung der Böden zu deuten sind. Es fällt ferner auf, wie Selaginella selaginoides und Botrychium lunaria, die in Rasen der subalpinen und alpinen Stufe verbreitet sind, oberhalb von $27 \mathrm{~cm}$ Profiltiefe steigende Werte aufweisen. Im Endabschnitt des kurzen Diagramms findet sich schließlich eine starke Zunahme der monoleten

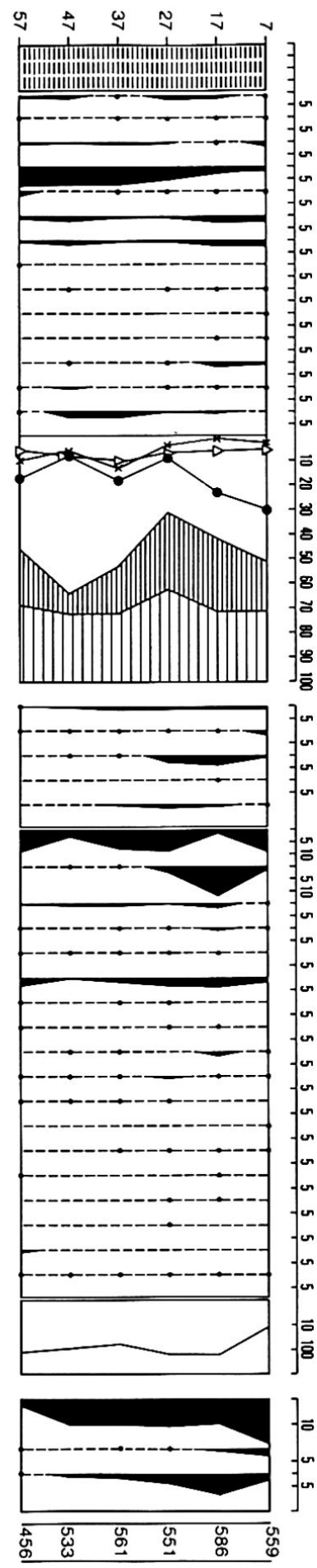

Tiefe (cm)
Stratigraphie
Juniperus
Salix
Betula
Alnus viridis
Alnus inc.lglut.
EMW
Quercus

Abb. 3: Pollendiagramm der fossilen Cyperaceentorfe des Lac du Plan

(Analyse und Entwurf des Diagramms von WEGMÜLLER) 
Farnsporen abgezeichnet. Die durchweg hohen Cyperaceen-Werte belegen die Ausbildung einer Verlandungszone, die wohl zur Torfbildung geführt hat.

Juglans tritt oberhalb von $27 \mathrm{~cm}$ Tiefe kontinuierlich auf, Carpinus setzt in $17 \mathrm{~cm}$ Tiefe nach oben hin ein. Beide Marken ergeben eine Zeitstellung, die nach unseren vegetationsgeschichtlichen Untersuchungen im Dauphiné (WEGMÜLLER, 1977) nicht vor 2500 B. P., vielleicht erst auf 2000 B. P. anzusetzen ist. Die Torfschichten unterhalb $27 \mathrm{~cm}$ Tiefe sind demzufolge ins Ältere Subatlantikum einzustufen, möglicherweise reichen sie noch ins späte Subboreal zurück. Das Radiokarbonalter von 1690 \pm 40 B. P. für die Torfprobe in $57 \mathrm{~cm}$ Tiefe (= Pr. 1795) ist also allem Anschein nach zu jung ausgefallen. Das palynologisch bestimmte Alter läßt sich aber gut mit dem radiometrisch erzielten Datum von $3920 \pm 45$ B.P. (= Pr. 1804) für das liegende Holz (Pinus cembra) vereinbaren.

Von mehreren Stämmen, die 1982 im Lac du Plan entdeckt worden sind, wurden Proben für dendrochronologische und dendroklimatologische Untersuchungen entnommen. Die Bearbeitung des Materials wurde an der Eidgenössischen Anstalt für das forstliche Versuchswesen in CH-8903 Birmensdorf vorgenommen. Leider führten die Arbeiten zu keinen Resultaten, bedingt durch die anatomischen Besonderheiten der Holzstruktur des Arvenholzes.

Für dendrochronologische und dendroklimatologische Zwecke ist Pinus cembra nicht sehr geeignet. Eine Ursache liegt sicher in dem Umstand, daß diese Art häufig an extrem ungünstigen Standorten steht. Die Jahrringe spiegeln oft diese für das Wachstum des Baumes schlechten Bedingungen wider. Die Synchronisation von historischem und fossilem Material ist meist schwierig oder wie bei dem vorliegenden Material sogar unmöglich. Die artspezifischen Unterschiede der Nadelhölzer und dadurch bedingte Schwierigkeiten hat BIRCHER (1982) in seiner Arbeit ausführlich beschrieben. Anhand des vorliegenden Materials soll hier auf die bei Pinus cembra auftretenden Probleme eingegangen werden.

Im Gegensatz zu Larix decidua und Picea abies, die beide einen regelmäßigen Wuchs aufweisen, zeichnet sich $P i$ nus cembra durch beträchtliche Schwankungen in den radialen Jahrringgrenzen aus. Zudem sind die Spätholzzonen auf wenige Zellreihen reduziert. Die Dichteunterschiede zwischen Frühholz und Spätholz sind bei Pinus cembra im Vergleich zu den beiden anderen Arten deutlich kleiner. Da bei Pinus cembra viele großlumige Harzkanäle mit dünnwandigen Epithelzellen auch an der Jahrringgrenze auftreten, werden die Zellen im ohnehin schon spärlich vorhandenen Spätholz aufgelockert. Zudem erreichen die Harzkanäle bei dieser Art mengenund flächenmäßig den größten Anteil. Dies führt bei der mehrmaligen densitometrischen Dichteerfassung derselben Probenstellung zu einer hohen artspezifischen Dichtevariation. Nach BIRCHERs Berechnungen (1982) liegt diese bei Pinus cembra bei 34 bis $41 \%$ gegenüber $L a$ rix und Picea, die Werte von 10 bis $14,5 \%$ nicht über- schreiten. Schon allein diese Zahlen weisen deutlich auf die Synchronisationsgüte von Arven hin. Das häufige Auftreten von großen Harzkanälen wirkt sich ebenfalls auf die Jahrringbreite aus. Die Schwankungen der Jahrringbreiten erreichen Werte von über $20 \%$ der totalen Breite. Mit aufnahmetechnischen Maßnahmen können diese Probleme nur wenig abgeschwächt werden.

Wie die fossilen Arven von zum Teil beträchtlichem Ausmaß in den Lac du Plan hineingeraten sind, ist nicht leicht zu erklären. Auf Grund der Tatsache, daß in den Lac du Plan keine Fließgewässer einmünden, können die Arvenstämme und -äste in ihn kaum eingeschwemmt worden sein. Eher in Frage kommt, daß die Stämme durch Lawinen von Süden her in das kleine Seebecken gerissen worden sind. Darauf könnte auch die Tatsache verweisen, daß einer der Arvenstämme auf seiner Vorderseite ausgeprägte Schurfspuren zeigte. In taschenartigen Stammhöhlungen fanden sich darin neben den bereits erwähnten Holzresten von Alnus spec., Salix spec. und teilweise verkohlten Holzbestandteilen bzw. Nüssen von Pinus cembra noch ein Aststück von Lonicera sp. sowie Samen von Ranunculus sect., Batrachium, Potentilla sp. und Lilaceae. Die verschiedenen ${ }^{14} \mathrm{C}$-Daten verweisen zudem darauf, daß die im Lac du Plan geborgenen Arven nicht durch ein einziges katastrophenartiges Ereignis wie einen Lawinenabgang oder auch nur während eines einzigen besonders harten Winters in den Lac du Plan gelangt sein können.

Nicht ganz eindeutig zu beantworten ist die Frage, ob die relativ hohe Baum- bzw. Waldgrenze im Bereich des Lac du Planim Jüngeren Atlantikum bzw. in der ersten Hälfte des Subboreal im Zusammenhang mit der besonderen Klimagunst der postglazialen Wärmezeit zu sehen ist, oder ob sie heute, ohne Beeinflussung des Menschen, eine ähnlich hohe Lage wie zu jener Zeit aufweisen würde. Auf Grund der von SCHWARZ berechneten rezenten potentiellen Baumgrenze von $2200 \mathrm{~m}$ ist davon auszugehen, daß dies nicht zutrifft und selbst ohne anthropogone Einflußnahme die heutige Wald- und Baumgrenze unter der des Atlantikums und Subboreals liegt. Belege für eine während des Klimaoptimums der postglazialen Wärmezeit höhere Lage der Baumgrenze haben sich auch für die Schweizer Alpen (GAMPER, SUTER, 1982, S. 112; FURRER et al. 1987, S. 76) und die französischen Nordalpen insgesamt (WEGMÜLLER, 1977, S. 163) ergeben.

Zudem sprechen die im nordöstlichen Pelvoux nur bis in Höhen von 2150-2170 m auftretenden Solitärbäume nicht dafür, daß im Untersuchungsgebiet die Baumgrenze heute bis $2300 \mathrm{~m}$ nach oben reicht. Geht man davon aus, daß die in $2300 \mathrm{~m}$ Höhe im Lac du Plan geborgenen fossilen Aren nicht im geschlossenen Waldverband, sondern vereinzelt oberhalb davon gewachsen sind, so müßte die natürliche Baumgrenze seit der zweiten Hälfte des Subboreals um mindestens $100 \mathrm{~m}$ abgesunken sein. Nimmt man jedoch an, daß die im Lac du Plan gefundenen fossilen Arven zu einem geschlossenen Wald gehörten, hätte sich die natürliche Baumgrenze seit der Mitte des Subboreals noch wesentlich stärker erniedrigt. 
Eine deutliche Klimaverbesserung im Zuge des Atlantikums hat JORDA (1987, S. 64, 65, 67) in den französischen Südalpen durch zahlreiche ${ }^{14} \mathrm{C}$-Datierungen im Zusammenhang mit fossilen Böden und stärker lehmig-kolluvialen Akkumulationen nachweisen können. Im ebenfalls noch weitgehend der postglazialen Wärmezeit und damit dem holozänen Klimaoptimum zuzuordnenden Subboreal (WEGMÜLLER, 1977, S. 133; HOLZHAUSER. SUTER, 1982, S. 6) soll nach JORDA (1987, S. 66) in den Nebenästen des Ubaye-Tales der französischen Südalpen eine Phase geringerer Akkumulation, ja sogar der Terrassierung gefolgt sein. Die von JORDA $(1987$, S. 68) dafür gegebene Begründung ist jedoch in sich widersprüchlich. Denn er postuliert für das Subboreal in den französischen Südalpen einmal eine auf abgesunkenen Temperaturen beruhende Intensivierung der Solifluktion, andererseits aber eine damit eigentlich unvereinbare Zunahme der fluviatilen Einschneidungsprozesse in den Tälern.

In der Belledonne gewonnene Ergebnisse deuten darauf hin, daß an der Obergrenze der montanen Stufe Schwemmkegel nicht nur im Atlantikum, sondern auch noch mitten im Subboreal um 3900 B. P. intensiv durch Grobablagerungen vermurt worden sind (HANNSS, 1982, S. 180, Tabelle 2). Diese in der Belledonne erzielten Erkenntnisse stünden im Einklang mit der Ansicht von DE BEAULIEU (1977, S. 291) und BURGA (1987, S. 77), daß es im Subboreal zu einem stärkeren Temperaturabfall bzw. einer deutlichen allgemeinen Klimaverschlechterung in den Alpen gekommen sei.

\section{Zusammenfassung}

Im nordwestlichen Pelvoux der französischen Nordalpen konnte M.COUTEAUX in der in $2140 \mathrm{~m}$ Höhe gelegenen Tourbière de la Muzelle pollenanalytisch eine komplette, nicht mehr gletscherüberfahrene Abfolge des gesamten alpinen Spät- und Postglazials von der Ältesten Dryas bis in das Subatlantikum nachweisen. Aus Berechnungen der spätglazialen Schneegrenzen ergibt sich im Zusammenspiel mit diesen pollenanalytischen Ergebnissen, daß auch im Pelvoux-Massiv, abgesehen vom Egesen-, Bocktentäli- und Kromer-Stand, alle älteren Gletschervorstöße des Spätglazials mit ziemlicher Sicherheit bereits vor dem Bölling stattgefunden haben.

Die heutige natürliche potentielle Baumgrenze im Bereich von Les Deux-Alpes liegt nach Berechnungen von R. SCHWARZ bei $2200 \mathrm{~m}$. Die reale Waldgrenze ist jedoch anthropogen hier meist mehrere hundert Meter unter die Höhenmarke von $2000 \mathrm{~m}$ herabgedrückt worden. Die starke, im wesentlichen wohl durch Abholzung und Überweidung hervorgerufene Erniedrigung der Waldgrenze äußert sich in bisweilen außergewöhnlich hohen Spannen zwischen der realen Wald- und Baumgrenze. Die 1982 von J.BIJU-DUVAL im Lac du Plan gemachten Holzfunde beweisen, daß den Radiokarbondatierungen gemäß zwischen 4800 B. P. und 3920 B. P., also im Jünge- ren Atlantikum und älteren Subboreal und damit während der postglazialen Wärmezeit, wenigstens Bäume, und zwar weitgehend Zirben (Pinus cembra), wenn nicht sogar Wälder, mindestens $100 \mathrm{~m}$ über der heutigen potentiellen Baumgrenze gestockt haben. Auf Grund anatomischer Besonderheiten der Holzstruktur des Arvenholzes konnten die geborgenen Holzscheiben von w. H. SCHOCH leider dendrochronologisch nicht näher ausgewertet werden. Auf das Vorherrschen der Arven an der Baumgrenze in den zentralen Teilen der Alpen nicht nur heute, sondern auch bereits in der postglazialen Wärmezeit verweisen die von S. WEGMULLER an Torfen des Lac du Planerzielten pollenanalytischen Ergebnisse. Die Torfe müssen nach ihm aus dem Älteren Subatlantikum bzw. dem jüngeren Subboreal stammen. Sie sind damit wesentlich älter, als es die Radiokarbondatierung der pollenanalytisch bearbeiteten basalen Torfe mit rund 1700 B. P. erwarten läßt. Demgegenüber steht das Radiokarbonalter von rund 4000 B.P. des Arvenstammes im Liegenden der Pollensequenz mit den pollenanalytischen Befunden in gutem Einklang.

\section{Anmerkungen}

1 Auf der Carte touristique 1:25 000, Massif des Ecrins findet sich dafür der Name Ruisseau de la Pisse. Im vorliegenden Artikel wird jedoch der von COUTEAUX (1983a, S. 39) benutzte Name Pisse de la Muzelle verwendet.

2 Die aufgeführten Klimawerte entstammen der Datensammlung von $\mathrm{CH}$. PEGUY (Grenoble) und wurden auf die Standardperiode 1931-1960 umgerechnet (Näheres HANNSS, 1982, S. 7)

3 Frau Münnich und Herrn Kromer sei herzlich für die Durchführung der Radiokarbondatierungen im Institut für Umweltphysik der Universität Heidelberg gedankt.

\section{Literatur}

DE BEAULIEU, J. L. (1977): Contribution pollenanalytique à I'histoire tardiglaciaire et holocène de la végétation des Alpes méridionales françaises. 0.0 .

DE BEAULIEU J. L., JORDA, M. (1977): Tardiglaciaire et Postglaciaire des Alpes de Haute Provence. Le glaciaire de la Blanche-Trois Evêchés. In: Bulletin de l'Association Française pour l'Etude du Quaternaire, S. 3-15.

BIRCHER, W. (1982): Gletscher und Klimageschichte des Saastales. Glaziologische und klimatologische Untersuchung. Physische Geographie Universität Zürich, 9.

BOREL, J.-L., JORDA, M., MONJUVENT, G. (1984): Chapitre II. Variations climatiques, morphogenèse et évolution de la végétation postwurmiennes dans les Alpes françaises. Les Alpes, $25^{\mathrm{e}}$ Congrès Internationale de Géographie, Paris, S. 43-53.

BURGA, C. (1987): 3. Vegetationsgeschichte seit der Späteiszeit. In: Geographica Helvetica, 42, S. 71-80.

BURGA, C. (1981): Das Pollendiagramm Crap Alv, 2045 m. In: Maisch M.: Glazialmorphologische und gletschergeschichtliche Untersuchungen im Gebiet zwischen Landwasserund Albulatal (Kt. Graubünden, Schweiz). Physische Geographie Universität Zürich, 3, S. 141-147. 
COUTEAUX, M. (1984): Présence, datages et signification phytosociologique de macro-restes de Pinus et de pollens de Pinus cembra L. à 2050 m dans le vallon de la Lavey (Massif des Ecrins, Isère, France). In: Paléobiologie, Genève, S. 55-62.

COUTEAUX, M. (1983a): Fluctuations glaciaires de la fin du Würm dans les Alpes françaises, établies par les analyses polliniques. In: Boreas, 12, S. 35-56.

COUTEAUX, M. (1983b): Géomorphologie et évolution phytogéographique tardiglaciaire et holocène aux Deux-Alpes (Isère, France): contribution pollenanalytique. In: Revue de Géographie Alpine, 71, S. 143-163.

COUTEAUX, M. (1983c): Déboisements d'altitude par les charbonniers de l'Oisans: contribution pollenanalytique. $108^{\circ}$ Congrès National des Sociétés Savantes, Grenoble, Philologie et Histoire, S. 69-87.

COUTEAUX, M. (1982a): La tourbière et le glacier de la Muzelle (Parc National des Ecrins). Travaux Scientifiques du Parc National des Ecrins, 2, S. 31-52.

COUTEAUX, M. (1982b): Récurrences glaciaires en Oisans au Tardiglaciaire. Société Hydrotechnique de France, Section de Glaciologie, Actes de la Réunion du 4-5 mars.

DELIBRIAS, G., GABERT, J., JORDA, M. (1984): Données nouvelles sur la chronostratigraphie et l'évolution paléomorphologique postglaciaire des Alpes françaises du Sud (Moyenne Durance). Comptes Rendus Hebdomadaires des Séances de l'Académie des Sciences, Paris, 299, Série II, S. 263-266.

EDOUARD, J. L. (1978): La glaciation du bassin de la Romanche. Contribution à l'étude des fluctuations postwürmiennes, Grenoble.

FURRER et al. (1987): Zur Gletscher-, Vegetations- und Klimageschichte der Schweiz seit der Späteiszeit. In: Geographica Helvetica, 42, S. 61-91.

GAMPER, M., SUTER, J. (1982): Postglaziale Klimageschichte der Schweizer Alpen. In: Geographica Helvetica, 37, S. 105-114.

HANNSS, Ch. (1984): La constitution des plaines alluviales et leurs rebords dans la vallée de l'Isère entre l'embouchure de l'Arc et le bassin de Moirans, ainsi que de la plaine alluviale de la Leysse dans le Val du Bourget. Revue de Géographie Alpine, 72, S. 439-456.
HANNSS, Ch. (1982): Spätpleistozäne bis postglaziale Talverschüttungs- und Vergletscherungsphasen im Bereich des Sillon alpin der französischen Nordalpen. Mitteilungen der Kommission für Quartärforschung der Österreichischen Akademie der Wissenschaften, 4.

HANNSS, Ch. (1980): Formenschatz und mutmaßliches Alter einer spätglazialen Moränenabfolge im inneralpinen Trokkengebiet der Vanoise (französische Nordalpen). Tübinger Geographische Studien, 80, S. 177-193.

HANNSS, Ch. (1973): Conséquences morphologiques de nouvelles datations au C-14 dans le sillon alpin près de Grenoble. In: Revue de Géographie Alpine, 61, S. 179-201.

HOLZHAUSER, H., SUTER, J. (Hrsg.) (1982): Exkursionsführer Teil B: Westschweiz. Hauptversammlung der Deutschen Quartärvereinigung in Zürich. Physische Geographie Universität Zürich, 7.

JORDA, M. (1987): Morphogenèse postglaciaire des régions intra-alpines françaises du Sud, le bassin de Barcelonnette (Ubaye) du Tardiglaciaire au Subboréal. Premières Communautées Paysannes en Méditerranée Occidentale sous la direction de J. Guilaine, J. Courtin, J.-L. Roudil, J.-L. Vernet, Paris, S. 1-69.

MAISCH, M. (1987): 2. Zur Gletschergeschichte des alpinen Spätglazials: Analyse und Interpretation von Schneegrenzdaten. In: Geographica Helvetica, 42, S. 63-71.

MAISCH, M. (1982): Zur Gletscher- und Klimageschichte des alpinen Spätglazials. In: Geographica Helvetica, 37, S. 93-104.

PATZELT, G., BORTENSCHLAGER, S. (1978): Zur Chronologie des Spät- und Postglazials im Ötztal und Inntal (Ostalpen, Tirol). In: FRENZEL, B. (Hrg.): Führer zur Exkursionstagung des IGCP-Projektes 73/1/24, Quaternary Glaciations in the Northern Hemisphere vom 5. bis 13.9.1976 in den Südvogesen, im nördlichen Alpenvorland und in Tirol. Bonn-Bad Godesberg, S. 185-197.

SCHWARZ, R. (1983): Simulationsstudien zur Theorie der oberen Baumgrenze. In: Erdkunde, 37, S. 1-11.

TONNEL, A., OZENDA, P. (1964): Séries de végétation de la moitié Sud du département de l'Isère, Documents pour la Carte de la Végétation des Alpes, 2, S. 9-36.

WEGMÜLLER, S. (1977): Pollenanalytische Untersuchungen zur spät- und postglazialen Vegetationsgeschichte der französischen Alpen. Bern. 